\title{
Inferiority vs. Superiority
}

\author{
By Stephan Guth \\ In preparation.
}

Evernote keywords: issues tagged for first-priority treatment in this entry

- Abla Fahita

- absurdity, tragedy $=$ comedy

- adab sākhir

- humo(u)r

- Ahmad Ragab

- irony

- 'Alī Galāl (Alycature)

- Almanawihly

- Anā Halūma

- Andeel (Qandīl)

- April Fools

- Bassem Youssef (Bāsim Yūsuf)

- Islām Ğāwīsh

- joke(s)

- making fun

- most shared cartoon on asa7bess in the period 25 June to 8 July 2016

- obey vs. defy elder

- Batman

- black humour

- breaking taboos

- caricature

- cartoon(s), cartoon soap opera

- Oum el Dounya (Umm il-dunyā)

- playfulness

- provocation

- Qandīl > Andeel

- Reality $=$ Joke (Satire), Reality $=$ Satire

- sarcasm

- challenging the givens, challenging stereotypes

- satire, adab sākhir, unintentional satire

- sense of humour

- social media satire

- comedy, comedy and drama production

- stand-up comedy

- stupidity of Ministers

- Comic Con

- condoms, condom video

- converse shoes

- Tawfīq 'Ukāsha

- tickling the mighty

- dark comedy

- dark present

- TV show Allo Jeddah

- unintentional satire

- derision

- vulgarity

- al-Waraqah 2

- exceptions

- government policy as satire

- What about the first Oscar?

- WillisFromTunis, Willis from Tunis

\section{Entries pointing here}

ARRAYs - Baby Milk $\downarrow$ Celebrities $\downarrow$ Clash $\downarrow$ Commemoration / Memorial Days $\downarrow$ Conversions Disappearances $\downarrow$ Dual Identities / Masking $\downarrow$ Father Figures $\downarrow$ Football $\downarrow$ Garbage $\downarrow$ Gated Communities / Compounds $\star$ Mobile Phones $\downarrow$ Self-help $\diamond$ Social Media $\downarrow$ The Suspect Foreigner Valentine's Day $\downarrow$ The Voice from Above

Codes - Beautiful vs. Ugly Male $v s$. Female $\downarrow$ Past $v s$. Present $\downarrow$ True $v s$. False

CODES COLLAPSED - True $=$ False $($ Life in Limbo $)$

\stephan.guth@ikos.uio.no

Journal of Arabic and Islamic Studies • 21 (2021) - Themed Section In2016: *318_*318 (C) Stephan Guth, Dept. of Culture Studies and Oriental Languages (IKOS), University of Oslo, NO 